\title{
3D Image Reconstruction from Exponential X-ray Projections: a Completeness Condition and an Inversion Formula
}

\author{
Frédéric Noo, Rolf Clackdoyle, Jean-Marc Wagner
}

\begin{abstract}
This work concerns the problem of reconstructing a 3D image from exponential $X$-ray (parallel-beam) projections. It is shown that exact reconstruction can be achieved when the projections are known on a set of directions $\Omega$ which satisfles the Orlov condition for nonattenuated projections. More specifically, it is shown that exact reconstruction can be achieved when the set $\Omega$ is intersected by every great circle on the unit sphere, provided the product $\mu R$ is sufficiently small, where $R$ is the radius of the region where the image is non-zero and $\mu$ is the attenuation coefficient. A reconstruction method is suggested and simulation results are provided to demonstrate the exactness and usefulness of the method.
\end{abstract}

Keywords - SPECT, attenuation correction, exponential parallel-beam projections, Orlov's condition

\section{INTRODUCTION}

This work concerns the mathematical problem of reconstructing a 3D image $f(\underline{x})$ from exponential X-ray (parallelbeam) projections. This problem is stated as follows:

Let $\underline{\theta}$ be some unit vector and let

$$
\begin{array}{r}
p(\underline{\theta}, u, v)=\int_{-\infty}^{\infty} d t f(u \underline{\alpha}+v \underline{\beta}+t \underline{\theta}) \exp (\mu t) \\
(u, v) \in \mathbb{R}^{2}
\end{array}
$$

be the exponential $X$-ray projection of $f(\underline{x})$ in the direction $\underline{\theta}$. Given $p(\underline{\theta}, \cdot, \cdot)$ for all vectors $\underline{\theta}$ in a subset $\Omega$ of the unit sphere, determine $f$.

Note that the vectors $\underline{\alpha}$ and $\beta$ in (1) are unit orthogonal vectors perpendicular to $\underline{\theta}$, while $u$ and $v$ are Cartesian coordinates used to specify different lines in the direction $\underline{\theta}$. The constant $\mu$ is the attenuation coefficient.

In the case where $\mu=0, p(\underline{\theta}, u, v)$ is a non-attenuated parallel-beam projection of $f$. The reconstruction theory for this situation has been widely covered in the literature. Of particular interest is the work of Orlov [1] who showed that exact reconstruction of $f(\underline{x})$ is possible when $\Omega$ is intersected by every great circle on the unit sphere.

When $\mu \neq 0$, reconstruction of $f$ from $p(\underline{\theta}, u, v)$ is more difficult and only particular geometries have been investigated [2]-[9]. From these works, it is known that exact reconstruction can be achieved when $\Omega$ is anyone of the following sets: a great circle $[2,3]$, the full sphere $[4,5]$, a union of great circles [6], a semi great circle [7], a semi

F. Noo and R. Clackdoyle are with the University of Utah (Department of Radiology). E-mail: noo@doug.med.utah.edu.

J-M Wagner is with the University of Liège (Department of Telecommunications). E-mail: wagner@montefiore.ulg.ac.be equatorial band [8] and a union of small circles satisfying Orlov's condilion (RSH-SPECT geonelry) [9].

In this work, we show that Orlov's condition is actually valid for reconstruction of $f(\underline{x})$ from $p(\underline{\theta}, u, v)$ when $\mu \neq 0$. More specifically, we show that exact reconstruction of $f(\underline{x})$ is possible when $\Omega$ is intersected by every great circle on the unit sphere provided the product $\mu R$ is sufficiently small, where $R$ is the radius of the region where $f(\underline{x})$ is non-zero.

Care must be taken with the definition of $\Omega$ in the case where $\mu \neq 0$. In the non-attenuated case where $\mu=0$, $p(\underline{\theta}, u, v)=p(-\underline{\theta}, u, v)$ and it is customary to assume that $\Omega$ is symmetric (i.e. if $\underline{\theta} \in \Omega$, then $-\underline{\theta} \in \Omega$ ). When $\mu \neq 0$, the reconstruction problem is different for symmetric and non-symmetric sets $\Omega$ because $p(\underline{\theta}, u, v) \neq p(-\underline{\theta}, u, v)$. The results in this paper apply to both types of sets.

This work finds its main application in SPECT imaging. Indeed, assuming that the attenuation is constant in the activity region, it is known that ideal SPECT data are related to exponential X-ray projections by a set of multiplicative weights defined from the attenuation map [10].

\section{METHOD}

Suppose that $\mu=0$. In this case, it is known that there exists a filter $h(\underline{\theta}, u, v)$ such that

$$
f(\underline{x})=\int_{\Omega} d \underline{\theta}\left(h * p_{\mu=0}\right)(\underline{\theta}, u=\underline{x} \cdot \underline{\alpha}, v=\underline{x} \cdot \underline{\beta}),
$$

where the symbol $*$ denotes a convolution operation.

Our inversion formula is based on the existence of the above filter $h(\underline{\theta}, u, v)$ and the relation

$$
f(\underline{x})=f_{0}(\underline{x})+(W * f)(\underline{x})
$$

where

$$
f_{0}(\underline{x})=\int_{\Omega} d \underline{\theta} \exp (-\mu \underline{x} \cdot \underline{\theta})(h * p)(\underline{\theta}, \underline{x} \cdot \underline{\alpha}, \underline{x} \cdot \underline{\beta}),
$$

and

$$
W(\underline{x})=\int_{\Omega} d \underline{\theta}(1-\exp (-\mu \underline{x} \cdot \underline{\theta})) h(\underline{\theta}, \underline{x} \cdot \underline{\alpha}, \underline{x} \cdot \underline{\beta}) .
$$

Let $\boldsymbol{R}$ be such that $f(\underline{x})=0$ for $|\underline{x}|>\boldsymbol{R}$ and let

$$
\chi(\underline{x})= \begin{cases}1 & \text { if }|\underline{x}|<R \\ 0 & \text { otherwise }\end{cases}
$$


In practice $R$ is always finite since $f$ is physically restricted to a finite region. Using $\chi$, the integral equation (3) can be rewritten in the form

$$
f=\chi f_{0}+\chi(W * f)=\chi f_{0}+K f
$$

where $K$ is an operator such that $K f=\chi(W * f)$. Because $W(\underline{x})$ tends to zero when $\mu$ tends to zero and $\chi(\underline{x})$ restricts the action of $W$ to the region $|\underline{x}|<R,\|K\|<1$ for $\mu R$ sufficiently small and equation (7) admits then the unique solution

$$
f=f_{0}+\sum_{l=1}^{\infty} \hat{K}^{l} \chi f_{0} .
$$

\section{Simulation}

We have implemented formula (8) for three different trajectories: a great circle, a semi-great circle, and the RSHSPECT geometry with a slant angle of $30^{\circ}$ and three detector positions separated by $60^{\circ}$. Figure 1 shows the results obtained from computer-simulated projections of a heart phantom made up of ellipsoids, with $20 \%$ activity in the ventricules. The attenuation coefficient was $\mu=0.0152 / \mathrm{mm}$ and the radius of the field-of-view was $R=74 \mathrm{~mm}$. For each geometry, there were 150 projections (each of $128 \times 128$ square pixels of side $1.2 \mathrm{~mm}$ ) and the reconstruction was performed on a grid of $128^{3}$ cubic voxels of side $1.2 \mathrm{~mm}$. The quality of the results demonstrates the validity of the method for practical data parameters.

\section{REFERENCES}

[1] S. S. Orlow, "Theory of three dimensional reconstruction. 1. Conditions of a complete set of projections.", Sov. Phys.Crystallogr., 20, 312-314, 1975.

[2] O. Tretiak and C. Metz, "The exponential Radon transform", SIAM J. Appl. Math., 39(2), 341-354, 1980.

[3] C. E. Metz and X. Pan, "A unified analysis of exact methods of inverting the 2D exponential Radon transform, with implications for Noise Control in SPECT", IEEE Trans. Med. Imag., 14(4), 643-658, 1995.

[4] I. A. Hazou, "Inversion of the exponential X-ray transform. I: Analysis", Math. Methods in the Applied Sciences, Vol. 10(10), 561-574, 1988.

[5] Y. Weng, G. L. Zeng and G. T. Gullberg, "Filtered backprojection algorithms for attenuated parallel and cone-beam projections sampled on a sphere", in Three-dimensional Image Reconstruction in Radiation and Nuclear Medicine, ed. P.Grangeat and J.-L. Amans (Dordrecht: Kluwer), 19-34, 1996.

[6] J.-M. Wagner and F. Noo, "Three-dimensional image reconstruction from exponential parallel-beam projections", IEEE Transactions on Nuclear Sciences, (to appear) June 2001

[7] F. Noo and J.-M. Wagner, "Image reconstruction in 2D SPECT with 180-degree acquisition" submitted to Inverse Problems.

[8] F. Noo, R. Clackdoyle, J.-M. Wagner, "Inversion of the 3D exponential X-ray transform for a semi equatorial band.", submitted to the Fully 3D meeting conference.

[9] J.-M. Wagner, F. Noo, R. Clackdoyle, "Exact inversion of the exponential X-ray transform for RSH-SPECT.", submitted to the Fully 3D mecting conference.

[10] A. Markoe, "Fourier inversion of the attenuated X-ray transform", SIAM J. Math. Anal., 15(4), 718-722, 1984.

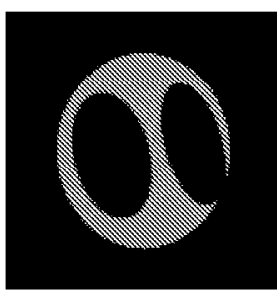

Great circle
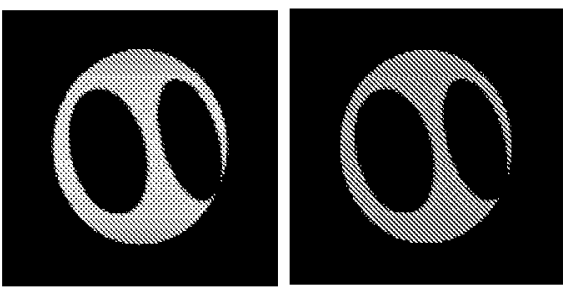

Semi great circle
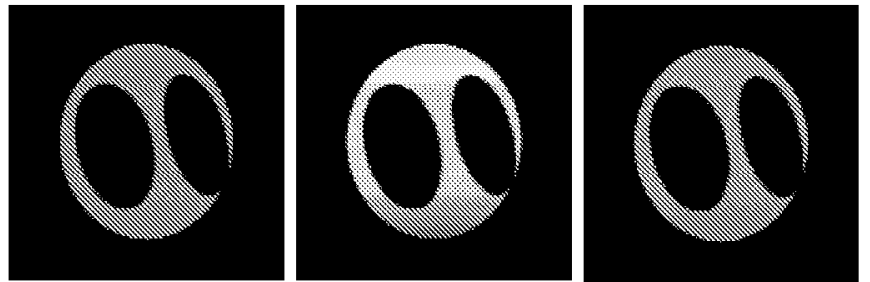

RSH-SPECT geometry
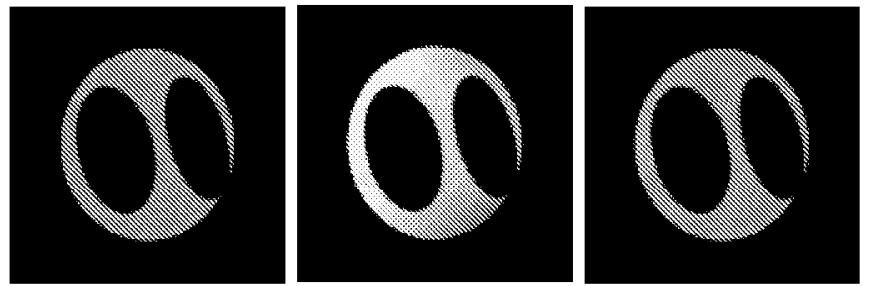

Fig. 1. Left column: reconstruction from non-attenuated data using (2). Middle column: reconstruction $f_{0}(\underline{x})$ (equation (4)). Right column: implementation of formula (8) with 4 terms. The images are displayed using the gray-scale $[0.8,1.2]$ window centered on the heart-wall activity value of 1. The average relative error for the reconstructions in the right column is approximately $3 \%$. Reconstruction time: about 5 min cpu per iteration on a SUN ULTRA 10. 